\section{Screening for deafness}

Eskander and Papsin ${ }^{1}$ may be somewhat disingenuous in their plea for neonatal screening for deafness. Underlying this notion is the unspoken concept that, having detected a hearing impairment in an infant, it is required that we treat him or her with a cochlear implant. Although early placement of cochlear implants may well foster acquisition of the spoken language of the larger community, as implied by the authors, it is incorrect to suggest that children with hearing impairments lack language skills per se.

The battle between the hearingimpaired community, which has a distinctive culture and a rich manual language, and a paternalistic hearing community, which feels the need to "fix" the "defective" individuals, has been ongoing for decades. Infants with hearing impairments babble with their hands, just as hearing infants do so orally. Given the opportunity, they then acquire language skills just as hearing infants do; it is simply a different language, expressed in a different mode.

This manual language reflects a culture foreign to the hearing community, and many in the hearing-impaired community argue that the insistence on cochlear implants is both unjustified, and, in the extreme, a form of cultural annihilation. I would suggest that the bland assumption that early detection is necessary and beneficial may not be as simple and obvious as Eskander and Papsin ${ }^{1}$ suggest.

\section{David Maxwell MD (retired)}

Middle LaHave, NS.

\section{Reference}

1. Eskander A, Papsin B. Screening infants for hearing impairment in Canada. CMAJ 2014;186:1048-9.

CMAJ 2015. DOI:10.1503/cmaj. 1150015

\section{The authors respond}

We understand why Maxwell ${ }^{1}$ may have felt that our screening advocacy article $^{2}$ insinuated that once a hearing impairment is detected in an infant, cochlear implantation is required. How- ever, this was never mentioned in the commentary, nor is it our practice.

We acknowledge that the hearingimpaired community has a rich culture and language. Screening is only meant to identify infants at a time when intervention (including timely introduction of a manual mode of communication) is possible. This then provides parents with choices that would allow hearing rehabilitation (which includes both hearing aids and potentially cochlear implants) to integrate their children into the hearing community.

We often, in our cochlear implant program, encounter parents who wish to keep their children in the deaf community, and we fully respect this decision. These parents nonetheless appreciate having an understanding of their options. In some cases, having identified the hearing loss early allows families to focus on learning sign language and assisting their children with the development of manual language. However, in our clinical reality, this is the minority of parents.

\section{Antoine Eskander MD ScM, Blake Papsin MD MSc}

Department of Otolaryngology (Eskander), University of Toronto; Department of Otolaryngology (Papsin), The Hospital for Sick Children, Toronto, Ont.

\section{References}

1. Maxwell D. Screening for deafness [letter]. CMAJ 2015;187:357.

2. Eskander A, Papsin B. Screening infants for hearing impairment in Canada. CMAJ 2014;186:1048-9.

CMAJ 2015. DOI:10.1503/cmaj. 1150016

\section{Residency matching woes}

Chanchlani ${ }^{1}$ raises important concerns about the residency match. I empathize with the frustration that many students have regarding the "lack of a clear rubric on how (residency programs) select candidates"; most programs base their decisions on "some combination of factors such as personal statement, reference letters and interview, the weight of these components is unknown and applicants do not know how eventual matches are made." ${ }^{1}$ The con- fusion and anxiety regarding the practices employed may be related to the fact that resident selection committees must now make decisions based on little or no useful data.

A recent meta-analysis on the topic identified that the best predictors of physician performance are objective measures such as medical school grades and standardized examination scores. ${ }^{2}$ Reference letters, interviews, dean's letters, personal letters and research experience were all found to have weak or no association with subsequent physician performance. ${ }^{2}$ Canadian medical schools now provide little or no objective data to resident selection committees - only 3 of 17 institutions provide any objective data regarding academic performance. ${ }^{3}$

Residency selection committees must now base their decisions on data points that have little or no utility regarding which students become the best physicians. They must sort through the large number of subjective data points that are variably presented in a nonstandardized format by the 17 Canadian medical schools. ${ }^{3}$ It's no wonder that students are confused regarding how they should be measured during the match - programs are too! It's time for Canadian medical schools to make an evidence-based decision and start reporting objective measures of academic performance. Maybe then, students can focus on learning medicine, rather than worrying about getting the "best" research project, elective or reference letter to secure them a residency position.

\section{Matthew McInnes MD}

Associate Professor of Radiology, University of Ottawa, Ottawa, Ont.

\section{References}

1. Chanchlani N. Seeking a better (residency) match. CMAJ 2014;186:979-80.

2. Kenny S, McInnes M, Singh V. Associations between residency selection strategies and doctor performance: a meta-analysis. Med Educ 2013;47:790-800.

3. Robins JA, McInnes MD, Esmail K. What information is provided in transcripts and medical student performance records from Canadian medical schools? A retrospective cohort study. Med Educ Online 2014;19:25181.

CMAJ 2015. DOI:10.1503/cmaj. 1150017 